Provided for non-commercial research and educational use only. Not for reproduction or distribution or commercial use.

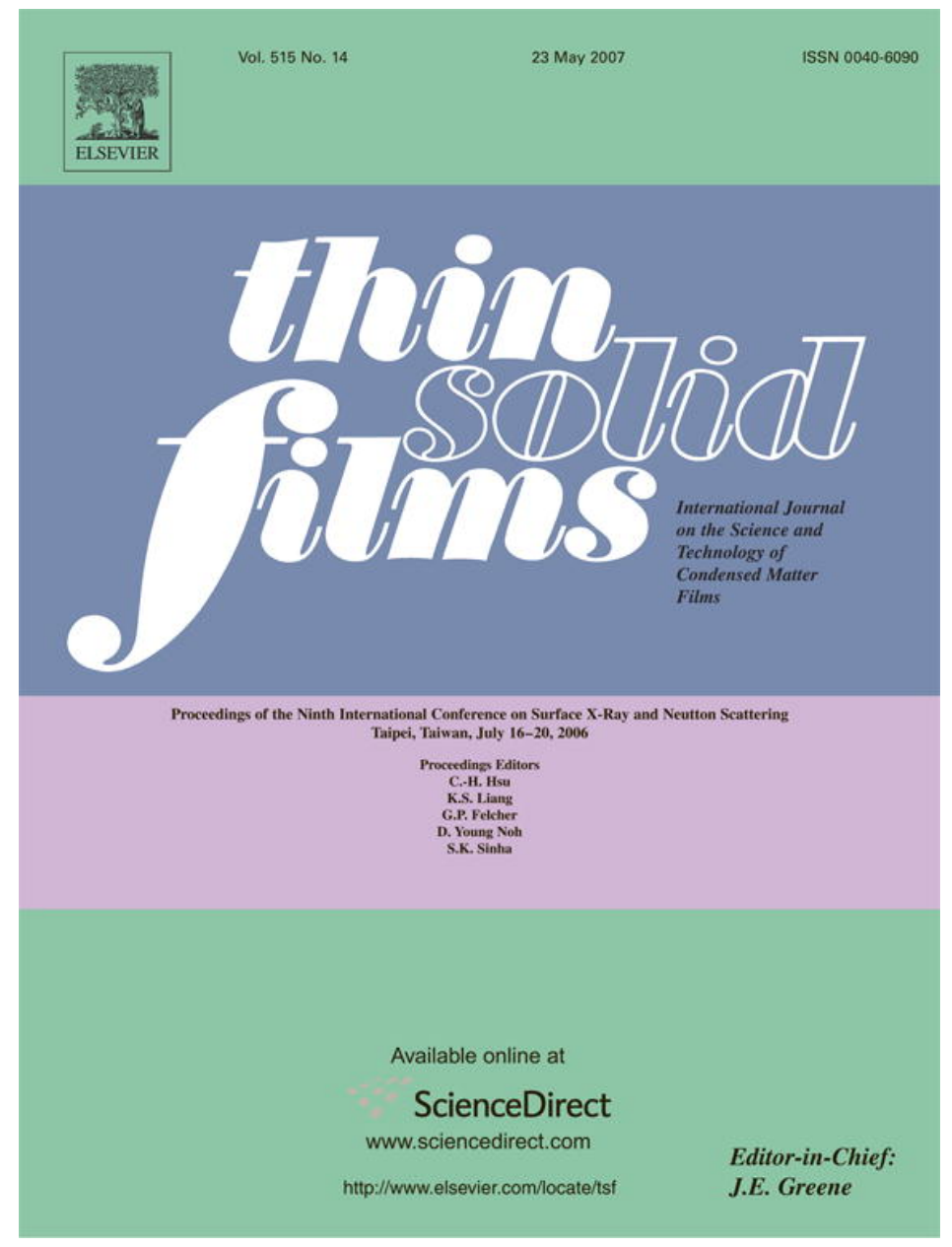

This article was originally published in a journal published by Elsevier, and the attached copy is provided by Elsevier for the author's benefit and for the benefit of the author's institution, for non-commercial research and educational use including without limitation use in instruction at your institution, sending it to specific colleagues that you know, and providing a copy to your institution's administrator.

All other uses, reproduction and distribution, including without limitation commercial reprints, selling or licensing copies or access,

or posting on open internet sites, your personal or institution's website or repository, are prohibited. For exceptions, permission may be sought for such use through Elsevier's permissions site at: 


\title{
Langmuir films of polycyclic molecules on mercury
}

\author{
L. Tamam ${ }^{\text {a }}$, H. Kraack ${ }^{\text {a, }}$, E. Sloutskin ${ }^{\text {a }}$, B.M. Ocko ${ }^{\text {b }}$, P.S. Pershan ${ }^{\text {c }, ~ M . ~ D e u t s c h ~}{ }^{\text {a,* }}$ \\ a Department of Physics, Bar-Ilan University, Ramat-Gan 52900, Israel \\ ${ }^{\mathrm{b}}$ Department of Soft Condensed Matter \& Materials Science, Brookhaven National Laboratory, Upton, NY 11973, USA \\ ' Department of Physics and DEAS, Harvard University, Cambridge, MA 02138, USA
}

Available online 27 January 2007

\begin{abstract}
Langmuir films (LFs) of biphenyl and anthracene derivatives on the surface of liquid mercury were studied by surface-specific X-ray and surface tension measurements. Phases of lying-down, side-lying and standing-up molecules were found, some of which exhibit long-range lateral order. The molecular symmetry and the position and nature of the side-, end-, and headgroups are shown to dominate the structural evolution of the LFs with surface coverage.
\end{abstract}

(C) 2007 Elsevier B.V. All rights reserved.

Keywords: Langmuir films; X-rays; Mercury

\section{Introduction}

Classical LFs are organic monolayers of amphiphilic molecules, spread on the surface of an aqueous subphase. They have been studied extensively since the end of the 19th century [1] due to their great interest for basic science and for technological applications. Their in-situ structure determination had to await the advent of synchrotron-based liquid surface X-ray methods, which emerged only in the early 1980s [2]. The first study, by these methods, of the surface-normal structure of water [3], was soon followed by the first in-situ structural studies of LFs on water [4]. Numerous X-ray studies of LFs of different organic molecules have been published during the two decades that have passed since then. All but very few used aqueous subphases. In view of the growing interest in LF-covered mercury electrodes in electrochemical studies [5], we have recently initiated a program of studying LFs on a liquid mercury subphase. By comparison with the same LF on water, the role of the subphase-LF interaction in determining the LF's structure can be assessed. Moreover, LFs of molecules which are either water-soluble, or non-spreading on water, can also be studied. Finally, the high surface tension of mercury $\left(\gamma_{\mathrm{Hg}} \approx 500 \mathrm{mN} / \mathrm{m}\right)$ results in a low surface roughness, $\sigma_{\mathrm{Hg}} \approx 1.4 \AA$. This allows

\footnotetext{
* Corresponding author.

E-mail address: deutsch@mail.biu.ac.il (M. Deutsch).

1 Present address: Infineon AG, 9500 Villach, Austria.
}

extending X-ray reflectivity (XR) measurements to a surfacenormal wavevector transfer of $q_{\mathrm{z}} \approx 3 \AA^{-1}$, four-fold higher than that achievable on aqueous subphases. A correspondingly higher resolution is achieved, allowing to study LFs as thin as 3-4 $\AA$.

Conjugate aromatic molecules are increasingly investigated as replacements for inorganic semiconductors ( $\mathrm{Si}, \mathrm{Ge}, \mathrm{GaAs}$ etc.), and as promising candidates for nano-sized and singlemolecule electronic devices [6]. The high charge mobilities of these molecules originate in their relatively large number of non-localized electrons. The molecular-scale structure of thin films of such molecules has been relatively scarcely studied, with almost all studies employing self-assembled layers (SAMs) on solid substrates. In SAMs, however, the epitaxy to the substrate's crystalline structure often dominates the monolayer's structure and its electrical properties. We present here a study of the structure of LFs of several types of conjugated aromatics on a liquid $\mathrm{Hg}$ subphase, which, being a liquid, can not impose a long-range order on the LF, but still preserves the metal-organic interaction of SAMs. Two classes of molecules have been chosen for the present study: biphenylthiols and anthracene and its derivatives. Their molecular structures are shown as insets in Fig. 1. The biphenyls were chosen since they have been well studied already as SAMs on longrange-ordered $\mathrm{Au}$ substrates [7]. When compared with the structure exhibited by the same molecules on a short-rangeordered, liquid $\mathrm{Hg}$ substrates, information could be obtained on 
the role of the substrate-monolayer interaction in the determination of the organic film's structure. The anthracene derivatives were chosen because of the acene molecules' importance for practical applications such as organic transistors, LEDs etc. [8].

\section{Experimental}

Surface pressure $(\pi) v s$. molecular area $(A)$ isotherms, and surface X-ray scattering, using synchrotron radiation, were employed in this study. A special mercury Langmuir trough was used, which allows measuring isotherm, and is also mountable on the liquid surface diffractometer for X-ray measurements. These were carried out at beamline X22B, NSLS, Brookhaven National Laboratory. The experimental and data analysis details have been published [9,11-15], and will not be repeated here.

\section{Results: biphenyls}

The two biphenyls studied, 4'-methyl-4-biphenyl-thiol (denoted MMB) and 4'-trifluoromethyl-4-biphenyl-thiol (denoted FMMB), differ only in their end-groups: the FMMB's has a dipole moment, while the MMB's does not. The crosssection of the two end-groups also differs. The thiol end-group provides a strong bonding to the mercury subphase for the surface-normal-aligned molecules [11].

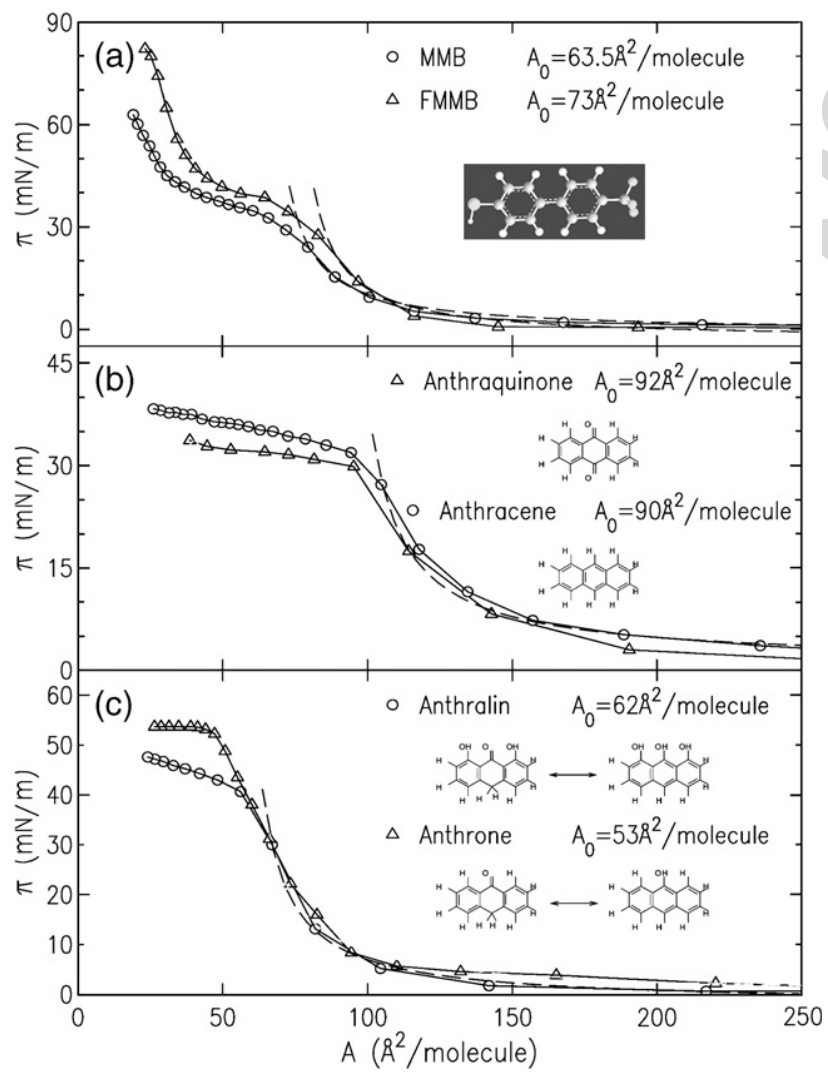

Fig. 1. The $\pi-A$ isotherms of the molecules listed. The dashed lines are fits by the Vollmer equation of an ideal hard-disc gas, $\pi\left(A-A_{0}\right)=k_{\mathrm{B}} T . A_{0}$ is the exclusion area, listed in the figure, due to the finite molecular size. The molecular structures are also shown. Arrows indicate keto-enol equilibria. The left side is the more stable keto form.
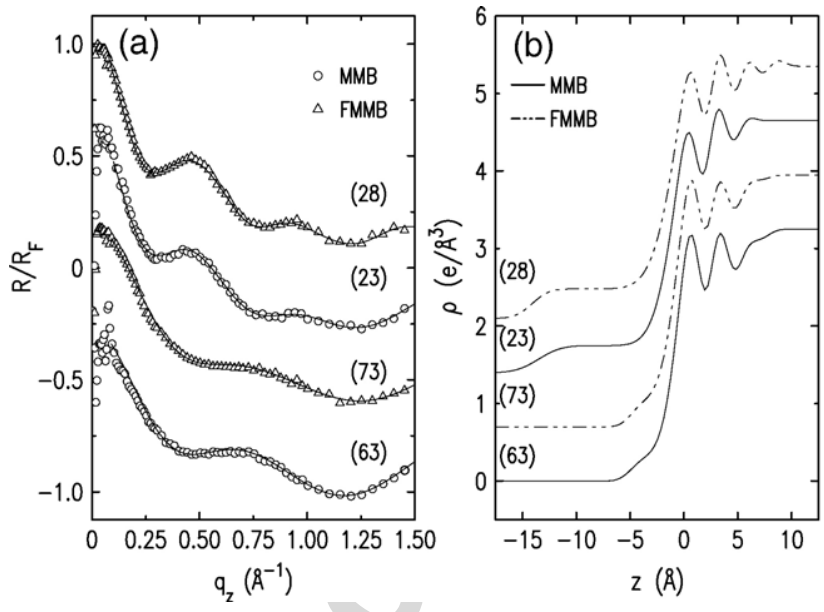

Fig. 2. (a) Measured Fresnel-normalized XR of MMB (circles) and FMMB (triangles) on mercury, with their box-model fits (lines). (b) The surface-normal electron density profiles derived from the fits in (a). Curves are shifted by 0.4 (a) and 0.7 (b) each for clarity. Numbers in parentheses are the nominal area per molecule.

\subsection{Isotherms}

The $\pi-A$ isotherms of MMB and FMMB on mercury, measured at $T=25^{\circ} \mathrm{C}$, are shown in Fig. 1(a).

Based on our previous studies [9-12], four regions can be identified: (a) For $A>100 \AA^{2} /$ molecule — a 2D gas of lyingdown molecules. (b) At $A \approx A_{0}$ - the onset of a single layer of closed-packed lying-down molecules. (c) The plateau is a coexistence between lying-down and standing-up molecules, with increasingly more of the molecules standing up with decreasing $A$. (d) The fast rising $\pi$ region at $A<40 \AA^{2} /$ molecule is a single layer of close-packed, standing-up molecules. These identifications are supported by the X-ray results discussed below. We note that the $A_{0}$ obtained for MMB is close to the $\sim 65 \AA^{2}$ calculated for the area of the lying-down molecule, using its $13.4 \AA$ length [7,16], and the $4.8 \AA$ average between the widths of a lying-down $(6.3 \AA)$ and a standing-up $(3.3 \AA)$ phenyl rings. This average is in line with the $90^{\circ}$-rotated conformation of the two phenyls, found here (see below) and on $\mathrm{Au}(111)$. The larger $A_{0}$ of the FMMB hints that its packing is not dominated by the phenyls, as indeed found in the X-ray data.

\subsection{Surface-normal structure}

The surface-normal structure was studied by XR measurements [17]. Fig. 2(a) shows a selection of the measured (circles) XR curves at the indicated coverages (normalized by the Fresnel XR of an ideally smooth and flat surface), along with their model fits (lines). The corresponding surface-normal density profiles are shown in Fig. 2(b).

The fits for both MMB and FMMB at $A \approx A_{0}$ yield a layer thickness of $d=4.9 \AA$ and an electron density of $\rho \approx 0.3 \mathrm{e} / \AA^{3}$. This $d$ agrees very well with the average of the diameter $(6.3 \AA)$ and thickness (3.3 $\AA$ ) of the phenyl ring [7], suggesting that one phenyl ring lies flat while the other is rotated by $\sim 90^{\circ}$ and 
stands on its side, a conformation found for the stripe phase of MMB on $\mathrm{Au}$ [7]. The fits at high coverage, $A=23$ and $28 \AA^{2} /$ molecule, for MMB and FMMB, respectively, yield $d_{\mathrm{MMB}}=$ $13.5 \AA$ and $d_{\mathrm{FMMB}}=14.1 \AA$ and $\rho_{\mathrm{MMB}}=0.35 \mathrm{e} / \AA^{3}$ and $\rho_{\mathrm{FMMB}}=$ $0.38 \mathrm{e} / \AA^{3}$. The lengths agree well with the extended "terminal$\mathrm{H}$ (or F)-to-Hg" length of a fully-extended molecule, indicating roughly surface-normal molecules. However, due to the cosine dependence of $d$ on the molecular tilt angle $\theta$ from the surface normal XR is not sensitive to small molecular tilts. Indeed, the grazing incidence diffraction (GID) measurements reveal such a tilt for MMB.

\subsection{Surface-parallel structure}

The surface-parallel structure was studied by GID and Bragg rod (BR) measurements [17]. GID peaks were found only for the standing-up phases, implying that only short-range lateral order may exists in the lying-down phase. Fig. 3(a) shows the GID and BR patterns measured for MMB at $A=30 \AA^{2} /$ molecule and $T=25^{\circ} \mathrm{C}$. The peaks observed the index as (11) and (02) in a two-molecule body-centered rectangular unit cell $5.56 \times$ $7.85 \AA^{2}$, yielding an X-ray-derived area $A_{\mathrm{x}}^{\mathrm{MMB}}=21.8 \AA^{2} /$ molecule. The 2:1 intensity ratio of the peaks, expected from this indexing, is indeed observed. $A_{\mathrm{x}}^{\mathrm{MMB}}$ is very close to that found for SAMs of MMB on $\mathrm{Au}$ [7]. The $5.56 \AA$ dimension is almost commensurate with the $3.18 \AA$ lateral inter-atomic distance of the mercury atoms at the surface: $5.56 / 3.18 \approx \sqrt{3}$. A
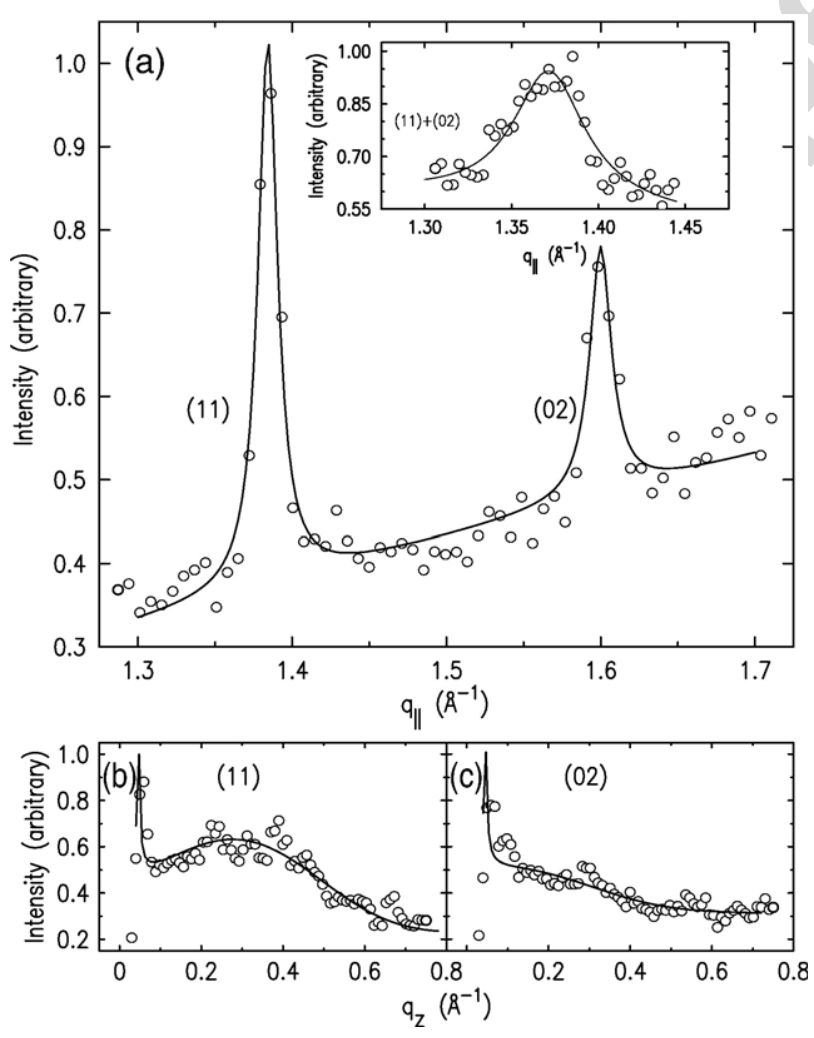

Fig. 3. The measured (circles) and the model-fitted (lines) (a) GID, and (b,c) BR, for MMB's standing-up phase at $T=25^{\circ} \mathrm{C}$. Inset to (a): the GID peak for the standing-up phase of FMMB. similar ratio was also found for LFs of alkyl-thiols on mercury [11]. The peaks' resolution limited widths imply, through the Debye-Scherrer formula [18], a lateral crystalline coherence length $\xi>1000 \AA$. This is a characteristic of the herringbonepacked crystalline CS phase of LFs on mercury $[9,11]$ and on water [1]. The BR and GID peaks' positions, 0.28 and $1.384 \AA^{-1}$ for (11), and 0 and $1.600 \AA^{-1}$ for $(02)$, yield a molecular tilt of $\theta=\arctan \left[0.28 /\left(1.384^{2}-1.600^{2} / 4\right)^{1 / 2}\right]=13.9^{\circ}$ [1] in the nearest-neighbor (NN) direction. A fit of the measured BR by a widely used model $[9,10,15]$, shown in lines in Fig. 5(b and $\mathrm{c}$ ), agrees well with the measured BRs and supports the conclusions drawn from the GID and BR peak positions.

For FMMB only a single GID peak is found (inset to Fig. 3(a), measured at $A=25.7 \AA^{2} /$ molecule and $T=25^{\circ} \mathrm{C}$ ). This indicates a hexagonal phase, with (rectangular) unit cell of $5.29 \times$ $5.29 \sqrt{3}{ }^{\circ} \mathrm{A}^{2}$ and $A_{\mathrm{x}}^{\mathrm{FMMB}}=24.2 \AA^{2} /$ molecule. The peak is much broader than those of $\mathrm{MMB}$, and yields a crystalline coherence length of only $110 \AA$. The BR (not shown) peaks at $q_{\mathrm{z}}=0 \AA^{-1}$, indicating untilted molecules. The small coherence length and the large $A_{\mathrm{x}}^{\mathrm{FMMB}}$ lead to the conclusion that this phase is a rotator phase, unlike the MMB's herringbone packing.

For further details on the structure of LFs of MMB and FMMB on mercury see [19].

\section{Interim discussion}

The key to the striking difference in the lateral structure of the crystalline LF phases of the apparently similar MMB and FMMB molecules, is the cross-sectional areas of the headgroups: $A_{\mathrm{CH}_{3}}=17.6 \AA$ and $A_{\mathrm{CF}_{3}}=25.4 \AA[19,20]$. Since $A_{\mathrm{CH}_{3}} \ll$ $A_{\mathrm{x}}^{\mathrm{MMB}}$, the biphenyl moiety [21] dominates the structure of $\mathrm{MMB}$, resulting in the herringbone packing, favored by this moiety. For FMMB $A_{\mathrm{CF}_{3}} \gg A_{\mathrm{x}}^{\mathrm{MMB}}$, inhibiting a similar packing. This results in a packing frustration, leading to the formation of a rotator, rather than a crystalline, order with a ten-fold lower $\xi$ than that of MMB. The frustration may be further aggravated by the close packing of the strong $\mathrm{CF}_{3}$-induced dipole moments of FMMB, which does not exist in MMB. For further details see $[19,20]$.

The lying-to-standing transition in both molecules can not be driven by surface pressure alone, since similar-length alkanes, for example, do not undergo such a transition with pressure [12]. The thiol headgroup must play an important role in this transition, as indeed found for alkyl-thiols [11]. This raises the possibility that other reactive moieties, in symmetric and asymmetric positions relative to the molecular axes, could also induce different structures in LFs. This hypothesis was explored in LFs of anthracene derivatives, which we discuss next.

\section{Results: symmetric anthracene derivatives}

\subsection{Isotherms}

Two molecules, anthracene and anthraquinone, having mirror symmetry with respect to both long and short axes of the molecule, were studied. Their $T=24{ }^{\circ} \mathrm{C}$ isotherms are shown in Fig. 1(b), with the molecular conformations shown as insets. The 
isotherms are similar to those of the biphenyls in Fig. 1(a), but lack the rising, low- $A$ part. The Vollmer-fitted $A_{0}$ values are very close to the area of a flat-lying anthracene molecule $A_{\text {calc }}=$ $l \times w \approx 12 \times 7.5=90 \AA^{2}$ calculated from its length $l$ and width $w$ [22]. The (slightly sloped) plateau at $A<90 \AA^{2} /$ molecule is, as for biphenyls, a coexistence region between two phases, the structures of which are obtained from the XR measurements.

\subsection{Surface-normal structure}

Fig. 4(a) shows Fresnel-normalized XR curves (open symbols), measured close to $\sim A_{0}$ and $\sim A_{0} / 2$ at $T=24{ }^{\circ} \mathrm{C}$. In the box-model fits at $A_{0}$ lying-down molecules were assumed, and $d$ had to be fixed at the known $4 \AA$ thickness of the molecule to avoid strong inter-parameter correlations. As observed in the figure, very good fits (lines) ensued, yielding $\rho=0.28$ and $0.29 \mathrm{e} / \AA^{3}$ for anthracene and for anthraquinone, which agree well with those calculated from the dimensions and number of electrons of the molecules. The $A_{0} / 2 \mathrm{XR}$ fits yielded $\rho=0.28 \mathrm{e} / \AA^{3}$ for both compounds, and $d=7.6$ and $8.8 \AA$, respectively, for anthracene and anthraquinone, reflecting the $\sim 1.3 \AA$ larger width of the anthraquinone due to its $\mathrm{OH}$ groups
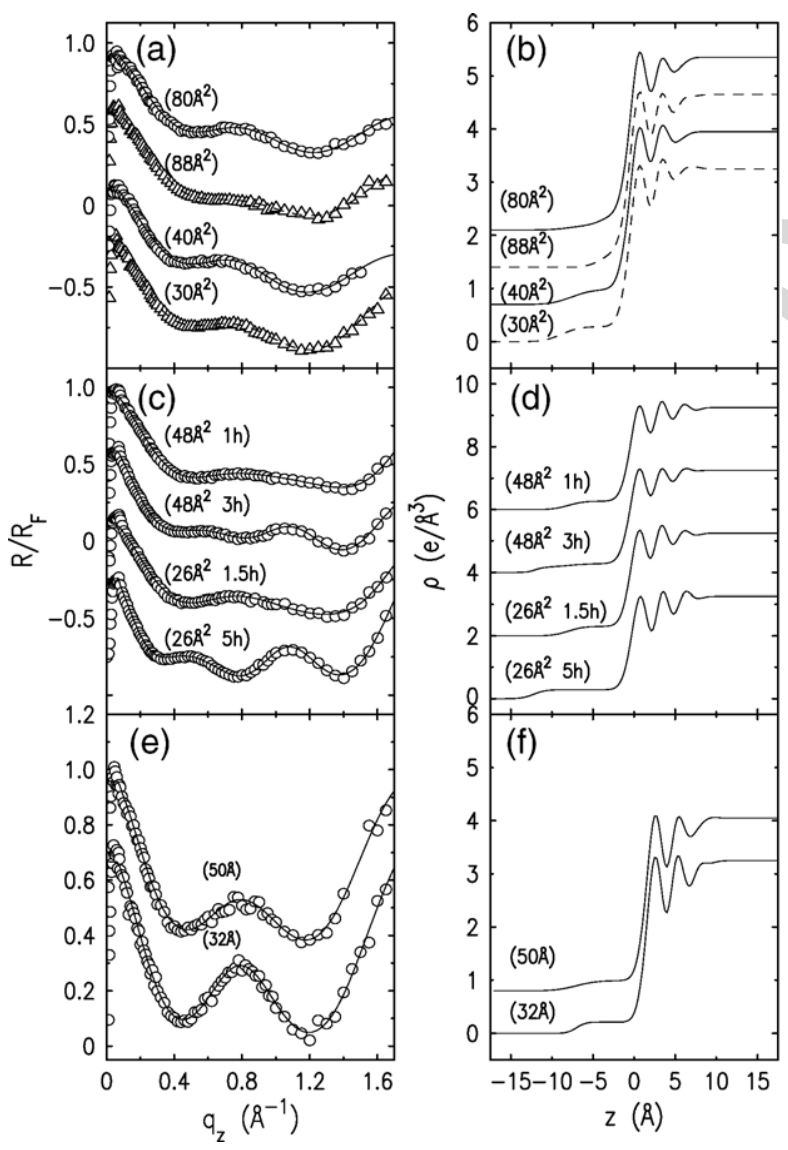

Fig. 4. Measured Fresnel-normalized XR curves (symbols), and box-model fits (lines), of LFs of (a) anthracene (triangles) and anthraquinone (circles), (c) anthrone, with time, in hours, since LF deposition, and (e) anthralin. The corresponding $\rho$ 's are shown in (b), (d) and (f). The nominal area per molecule for each curve is also given. Curves are shifted from each other for clarity. on both sides [23]. These $d$ values are close to the width of the anthracene molecule, implying that the molecules are sidelying, with surface-normal molecular planes and surfaceparallel long axes. These $d$ values could correspond, in principle, also to a bilayer of lying-down molecules. However, this would entail observing a corresponding mono- to bi-layer transition feature in the isotherm, found, e.g., for alkanes [12] and alcohols [9], which is not observed here.

Finally, no GID peaks were observed for both compounds at any $A$, indicating that no long-range lateral order exists in these LFs.

\section{Results: asymmetric anthracene derivatives}

\subsection{Isotherms}

The $T=24{ }^{\circ} \mathrm{C}$ isotherms of LFs of two asymmetric anthracene derivatives, anthrone and anthralin, are shown in Fig. 1(c), along with their molecular structure. Unlike other LFs on mercury, the Vollmer equation (dashed line in Fig. 1(c) for anthralin) yields $A_{0}$ values significantly smaller than the area of the flat-lying molecule, $A \gtrsim 90 \AA^{2} /$ molecule. The molecular plane is, therefore, normal to, or, at least, strongly tilted from, the surface plane.

\subsection{Surface-normal structure}

Fig. 4(c and e) shows Fresnel-normalized XR curves (open circles), measured at $T=24{ }^{\circ} \mathrm{C}$ at the listed $A$ and time after deposition, for LFs of anthrone (c) and anthralin (e). As for the symmetric derivatives, $\mathrm{XR}$ curves were measured close to $\sim A_{0}$ and $\sim A_{0} / 2$. The box-model fits (lines) yield the corresponding $\rho(z)$ profiles in Fig. 4(d and f).

For anthrone a time-dependent behavior was found [24]. The low-coverage, short-time $(1 \mathrm{~h})$ fits yield $d=8 \AA$, and $\rho=0.28$. With the anthracene molecule's dimensions [22], $12 \times 7.5 \times$ $3.88 \AA^{3}, d$ is close to the width of the molecule, indicating that the molecules are side-lying. A time-evolution into a thicker layer, indicated by a shorter XR modulation period, is found $3 \mathrm{~h}$ after LF deposition. A fit of this XR by a model accounting for coexisting side-lying and standing-up molecules (line) $[10,11,15,24]$ indeed reveals a $58 \%$ surface coverage by standing-up molecules $(d=12.2 \AA)$ coexisting with side-lying $(d=8 \AA)$ molecules. At $\sim A_{0} / 2$ a qualitatively similar timedependent behavior is found, except that a $100 \%$ coverage by the standing-up phase ensues at long times.

The XR curves of anthralin (Fig. 4(e and f)) yield $\rho=0.19$ and $0.21 \mathrm{e} / \AA^{3}$ at low and high coverage, respectively, and $d=8.9 \AA$ at both. The last datum implies that the molecules do not stand up even at high coverage.

\subsection{Surface-normal structure}

Unlike the symmetric compounds, anthralin and anthrone exhibit long-range lateral order, albeit only at high coverage. The crystalline packing of the two are, however, different and will be discussed separately. 

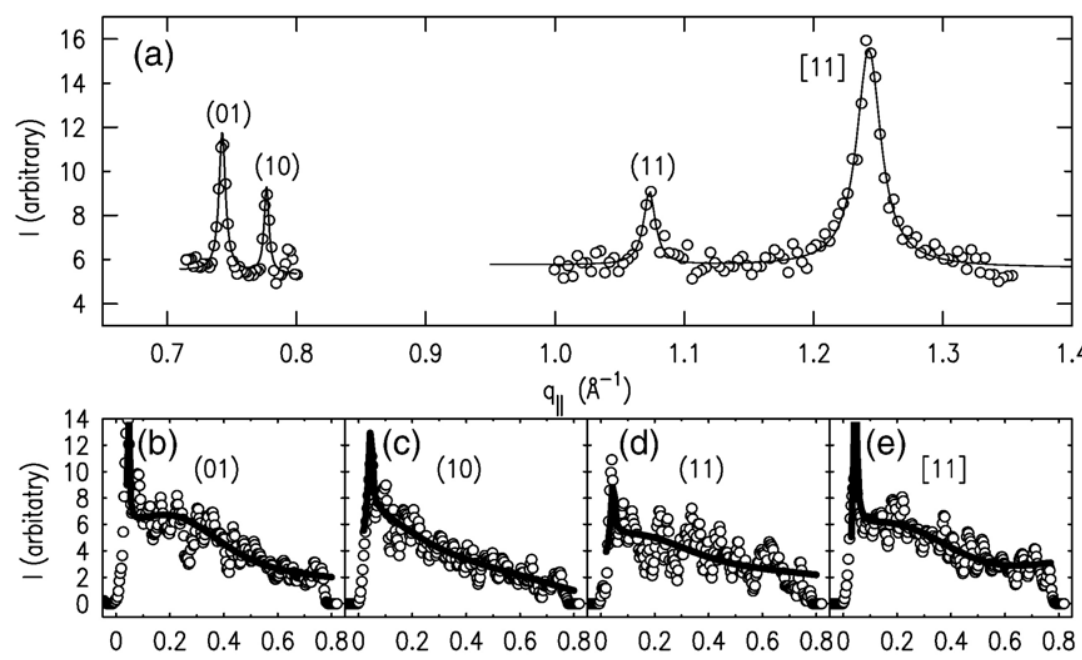

$\mathrm{q}_{2}\left(\AA^{-1}\right)$
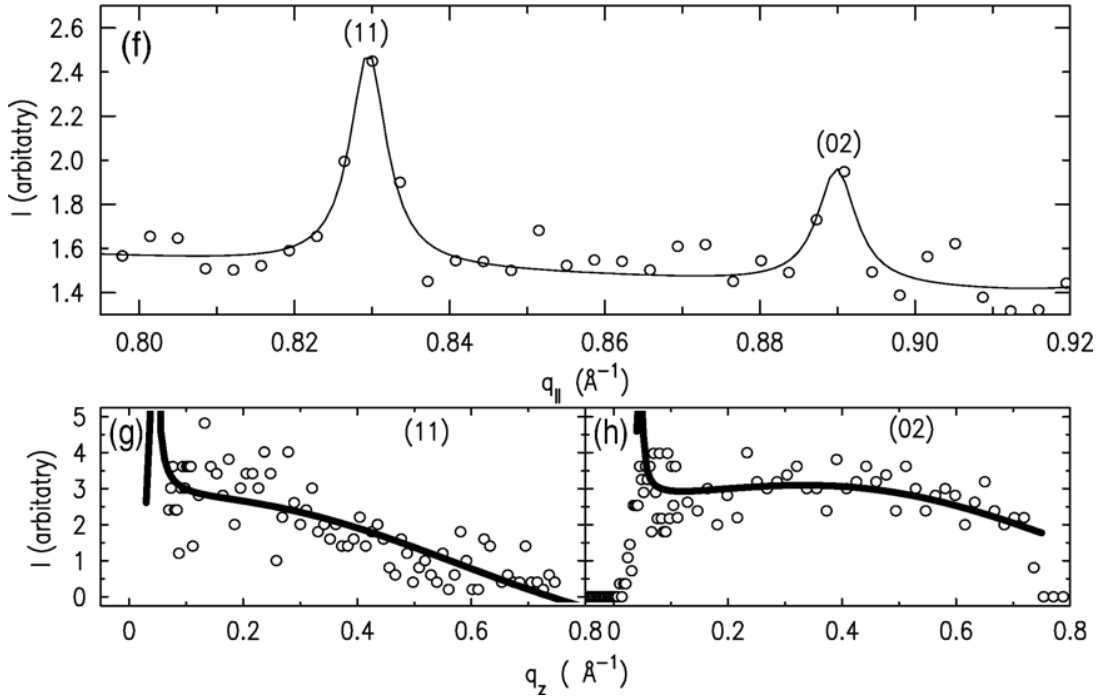

Fig. 5. Measured (symbols) and model-fitted (lines) GID patterns of anthrone (a) and anthralin (f) and the corresponding BRs, (b-e) and (g-h). In (a), ( $h k$ ) and $[h k]$ denote the rectangular and the hexagonal phases, respectively.

\subsubsection{Anthrone}

GID peaks for anthrone were found only at low temperatures and $A<32 \AA^{2} /$ molecule. These are shown in Fig. 5(a) (GID) and (b-e) (BR), measured at $A=26 \AA^{2} /$ molecule and $T=7.5^{\circ} \mathrm{C}$. No single unit cell allowing indexing of all four GID peaks could be found. However, the three lowest- $q_{\|}$peaks index in a two-molecule, non-centered rectangular unit cell [1] $\mathbf{a} \times \mathbf{b}=$ $8.09 \times 8.45 \AA^{2}$, yielding $A_{\mathrm{x}}=34.2 \AA^{2}$. This exceeds considerably the calculated area of a standing-up anthracene molecule [22], $A_{\text {calc }} \approx 3.8 \times 7.5=28.5 \AA^{2}$, indicating a possible tilt. The model fits (lines) to the measured BRs (symbols) in Fig. 5(b, c and d) indeed reveal a molecular tilt of $\theta=28^{\circ}$, in the nextnearest-neighbors (NNN) direction, b. However, even in the plane normal to the molecular long axis the molecular area, $A_{\perp}=\mathbf{a} \times \mathbf{b} \times \cos \theta=30.2 \AA^{2}$, is still larger than that of anthracene. The highest- $q_{\|}$GID peak in Fig. 5(a) had to be assigned to a coexisting hexagonal phase of a (rectangular) unit cell $\mathbf{a} \times \mathbf{b}=$ $5.84 \times 5.84 \sqrt{3} \AA^{2}$, with $A_{\mathrm{x}}=29.5 \AA^{2} /$ molecule. The modelfitted measured BR yields a small, $\theta=(7 \pm 3)^{\circ} \mathrm{NNN}$ tilt. The two coexisting phases differ greatly in their crystalline coherence lengths, as derived from the peaks' widths: $\xi>1000 \AA$ for the rectangular phase, and only $\xi \sim 200 \AA$ for the hexagonal phase. The physical reason for the coexistence of these phases is not clear.

\subsubsection{Anthralin}

The measured GID peaks in Fig. 5(f) are indexed as (11) and (02) in a two-molecule, centered rectangular unit cell $\mathbf{a} \times \mathbf{b}=8.97 \times 14.15 \AA^{2}$. The resultant $A_{\mathrm{x}}=63.46 \AA^{2} /$ molecule is close to $A_{0}$ (Fig. 1(c)). The fit (lines) of the two measured (symbols) BRs in Fig. 5(g and $\mathrm{h}$ ) yields a NNN molecular tilt of $\theta \approx 26^{\circ}$

The values of $\mathbf{b}$, and $d$, both significantly larger than the length (12 $\AA$ ) and width $(7.5 \AA)$ of the anthracene moiety [22], lead us to suggest tentatively that the long molecular axis of the side-lying molecule is tilted up from the surface. An uptilt of $\sim 20^{\circ}$ leads to a surface-projected molecular length of $\sim \sqrt{7.5^{2}+12^{2}}=$ $14.15^{\circ} \mathrm{A}$, and an $\sim 9.2 \AA$ average between the highest and 
lowest points of the molecule's top, close to the XR-derived $d=8.9 \AA$. The molecular packing found here is close to the packing found in the bulk crystal of anthralin [25]. The resolutionlimited GID peak widths indicate a large $\xi>1000 \AA$.

Further details of the structure of the anthracene derivatives' LFs will be given elsewhere [24].

\section{Discussion and conclusions}

The lying-down to side-lying (or to standing-up) transition seems to be a central motif in the phase behavior of the LFs studied here, and also in other LFs on mercury [10,11,15]. While lying-down maximizes the molecule-subphase contact, side-lying or standing-up maximizes the overlap between the $\pi$ bonds of adjacent molecules. The balance between these two free-energy-reducing tendencies largely determines the structure of the LF at any given $A$. We note in passing that while no lying-down phases were observed for anthrone and anthralin at $A \lesssim A_{0}$, it is likely that such phases exist at $A>A_{0}$.

The balance discussed above is further modulated by other interactions, induced by the head-, side-, or end-groups of the molecules comprising the LF. The strongly mercury-affine thiol headgroup induces a standing-up, rather than a side-lying, orientation in the biphenyls, and the LF's packing is further influenced by the end-group's size. By contrast, of the anthracene derivatives, none of which has a mercury-affine headgroup, only one exhibits a standing-up phase. The fact that the anthrone and the anthralin are already side-lying just below $A_{0}$, rather than lying-down as anthracene and anthraquinone, may have to do with another interaction, that between their strong molecular dipole moments $(3.3 \mathrm{D}$ and 3.6 D, respectively $[23,24])$, induced by the asymmetrically-attached $\mathrm{O}$ and $\mathrm{OH}$ moieties. The resultant dipole-dipole interactions can be minimized by an antiparallel dipole alignment, which requires the molecular planes to be parallel, i.e. side-lying, rather than lying-down. These are just a few qualitative examples. A more complete discussion is outside the scope and length limit of this paper. Moreover, such a discussion is strongly hampered by the scarcity of Å-resolution structural studies of LFs of additional molecules on mercury. Further studies, now underway in our laboratory, may soon fill some of the gaps and provide a better understanding of the physics underlying these films.

\section{Acknowledgements}

We thank A. Ulman and E. Ofer for contributions to this work. Support to P.S.P by the NSF (Grant No. DMR-0124936) and beamtime at X22B, NSLS, Brookhaven National Laboratory, are gratefully acknowledged. BNL is supported by U.S. DOE Contract No. DE-AC02-98CH10886.

\section{References}

[1] V.M. Kaganer, H. Möhwald, P. Dutta, Rev. Mod. Phys. 71 (1999) 779.

[2] J. Als-Nielsen, F. Christenson, P.S. Pershan, Phys. Rev. Lett. 48 (1982) 1107; P.S. Pershan, J. Als-Nielsen, Phys. Rev. Lett. 52 (1984) 759.

[3] A. Braslau, M. Deutch, P.S. Pershan, A.H. Weiss, J. Als-Nielsen, J. Bohr, Phys. Rev. Lett. 54 (1985) 114.

[4] S. Grayer Wolf, L. Leiserowitz, M. Lahav, M. Deutsch, K. Kjaer, J. AlsNielsen, Nature 328 (1987) 63;

P. Dutta, et al., Phys. Rev. Lett. 58 (1987) 2228;

Ch. Kjaer, et al., Phys. Rev. Lett. 58 (1987) 2224.

[5] D. Cahen, G. Hodes, Adv. Mater. 14 (2002) 789;

A. Salomon, et al., J. Am. Chem. Soc. 126 (2004) 11648;

M. Cohen Atiya, A. Nelson, D. Mandler, J. Electroanal. Chem. 593 (2006) 227.

[6] B.A. Mantooth, P.S. Weiss, Proc. IEEE 91 (2003) 1785; J. Jortner, M. Ratner (Eds.), Molecular Electronics, Blackwell, Oxford, 1997.

[7] T.Y.B. Leung, P. Schwartz, G. Scoles, F. Schreiber, A. Ulman, Surf. Sci. 458 (2000) 34.

[8] G. Maltezos, R. Nortrup, S. Jeon, J. Zaumseil, J.A. Rogers, Appl. Phys. Lett. 83 (2003) 2067.

[9] H. Kraack, B.M. Ocko, P.S. Pershan, E. Sloutskin, L. Tamam, M. Deutsch, Langmuir 20 (2004) 5386.

[10] H. Kraack, B.M. Ocko, P.S. Pershan, M. Deutsch, Science 298 (2002) 1404 H. Kraack, M. Deutsch, B.M. Ocko, P.S. Pershan, Nucl. Instrum. Methods Phys. Res., B Beam Interact. Mater. Atoms 200 (2003) 363.

[11] B.M. Ocko, H. Kraack, P.S. Pershan, E. Sloutskin, L. Tamam, M. Deutsch, Phys. Rev. Lett. 94 (2005) 017802.B.M. Ocko et al. (to be published).

[12] H. Kraack, B.M. Ocko, P.S. Pershan, E. Sloutskin, M. Deutsch, J. Chem. Phys. 119 (2003) 10339.

[13] H. Kraack, B.M. Ocko, P.S. Pershan, L. Tamam, M. Deutsch, J. Chem. Phys. 121 (2004) 8003.

[14] B.M. Ocko, X.Z. Wu, E.B. Sirota, S.K. Sinha, O. Gang, M. Deutsch, Phys. Rev., E 55 (1997) 3164.

[15] H. Kraack, B.M. Ocko, P.S. Pershan, E. Sloutskin, L. Tamam, M. Deutsch, Langmuir 20 (2004) 5375.

[16] W. Azzam, C. Fuxen, A. Birkner, H.T. Rong, M. Buck, Ch. Wöll, Langmuir 19 (2003) 4958.

[17] J. Als-Nielsen, D. McMorrow, Elements of Modern X-Ray Physics, Wiley, New York, 2001.

[18] A. Guinier, X-Ray Diffraction, Freeman, San Francisco, 1968.

[19] L. Tamam, H. Kraack, E. Sloutskin, B.M. Ocko, P.S. Pershan, A. Ulman, M. Deutsch, J. Phys. Chem., B 109 (2005) 12534.

[20] H.I. Kim, T. Koini, T.R. Randall, S.S. Perry, Langmuir 13 (1997) 7192.

[21] G. Casalone, C. Mariani, A. Mugnoli, M. Simonetta, Mol. Phys. 15 (1968) 339.

[22] C. Lane, A. Wise, Polycyclic Aromatic Hydrocarbon Structure Index, NIST, vol. 922, 1997.

[23] H.D. Flack, Philos. Trans. R. Soc. Lond., A 266 (1970) 561; P.A. Reynolds, Acta Crystallogr., A 31 (1975) 80.

[24] L. Tamam, H. Kraack, E. Sloutskin, B.M. Ocko, P.S. Pershan, E. Ofer, M. Deutsch, J. Phys. Chem. C 111 (2007) 2573; ibid. 2580.

[25] F.R Ahmed, Acta Crystallogr., B 38 (1980) 3184. 